\title{
Substructures of Gas-Ion-Irradiation-Induced Surface Blisters in Silicon Studied by Cross-Sectional Transmission Electron Microscopy
}

\author{
Shunsuke Muto and Naruaki Enomoto \\ Department of Materials, Physics and Energy Engineering, Graduate School of Engineering, \\ Nagoya University, Nagoya 464-8603, Japan
}

Internal structures of surface blisters and their precursors in $\mathrm{Si}$ formed by $\mathrm{H}^{+}, \mathrm{D}^{+}$and $\mathrm{He}^{+}$irradiation were examined by cross-sectional transmission electron microscopy (XTEM). The skin structures of $\mathrm{H}^{+}-\mathrm{D}^{+}$- and $\mathrm{He}^{+}$-blisters reflected the difference in the damage accumulation, and chemical interaction between the implant ion and silicon. These differences had a direct influence on the defect structures of the damaged layer, which played essential roles in the blistering mechanisms. It was found that blister skin thickness derived by grazing incidence electron microscopy (GIEM) and electron energy-loss spectroscopy (EELS) was underestimated compared to direct measurement by XTEM. The origin of this discrepancy is discussed.

(Received July 21, 2005; Accepted August 22, 2005; Published October 15, 2005)

Keywords: blister, silicon, cross-sectional transmission electron microscopy, plastic deformation, ion irradiation, electron energy-loss spectroscopy, hydrogen, helium

\section{Introduction}

Blistering phenomena have been widely observed in metal surfaces accumulating a high concentration of gas molecules through irradiation or nuclear transmutation. ${ }^{1,2)}$ Plastic deformation associated with blistering eventually evolves into flaking, a serious type of material degradation in nuclear environments. It is hence important to study the substructure of blister skins and blister precursors underlying the material surfaces of interest, and to elucidate the blistering mechanisms. One of the most commonly used methods to observe blistering phenomena has been scanning electron microscopy (SEM) ${ }^{3-7)}$ which enables observation of surface topography with relatively high spatial resolution, but cannot be used to explore the substructure of blisters.

The present authors developed a so-called grazing incidence electron microscopy (GIEM) technique for nondestructive structural analysis of surface protrusions, ${ }^{8)}$ and have used it successfully to characterize surface blistering phenomena in non-ductile materials such as $\mathrm{Si}^{9-11)}$ and SiC. ${ }^{12)}$ The method is particularly suitable for structural analysis of blisters because of their hollow structure with thin outer walls, transparent for the incident electron. We also employed an electron energy-loss spectroscopy (EELS) instrument equipped with a transmission electron microscope $(\mathrm{TEM})^{8-13)}$ to determine, not only the blister skin structure, but also the skin thickness and chemical composition. This method revealed the structural differences between blisters formed on $\mathrm{Si}$ and $\mathrm{SiC}$ surfaces irradiated by $\mathrm{H}^{+}, \mathrm{D}^{+}$and $\mathrm{He}^{+}$. In the case of $\mathrm{Si}$, the skins of blisters induced by $\mathrm{H}^{+}, \mathrm{D}^{+}$, and $\mathrm{He}^{+}$irradiation were single-crystalline, polycrystalline, and amorphous, respectively. In the case of $\mathrm{SiC}$, however, blister skins were amorphous, irrespective of the ion species used for irradiation. ${ }^{12)}$ This reflects the balance between the energy imparted to the surface by the implanted species (energy deposition) and the amorphization tendency of the target materials, key factors for elucidating the blistering mechanism of brittle materials. GIEM has been successfully used in observing well-developed surface blisters, however, it is necessary to examine the damage evolution underneath the sample surface to reveal the initial stage of blistering processes. This can only be realized by cross-sectional transmission electron microscopy (XTEM) of samples irradiated at doses below the blistering threshold.

We have already reported XTEM observations of blisters and their precursors in SiC. ${ }^{13)}$ In the present study, we present the XTEM results for Si. We also discuss the discrepancy between blister skin thickness estimations by GIEM-EELS and XTEM.

\section{Experimental Procedures}

The samples were prepared from $\langle 100\rangle$-oriented Si wafers that were electrochemically polished on one side. The wafers were cut into rectangular parallelepiped blocks $(2 \times 1 \times$ $1 \mathrm{~mm}$ ). Each block was mounted on a single-hole grid with the polished side edge-on and directed to the center of the grid. The polished side of the sample was then irradiated with $\mathrm{H}^{+}, \mathrm{D}^{+}$or $\mathrm{He}^{+}$ions at room temperature. The ion energies were selected to be 13,10 , and $16 \mathrm{keV}$ for $\mathrm{H}^{+}, \mathrm{D}^{+}$, and $\mathrm{He}^{+}$ irradiation, respectively, to provide nearly the same projected range of implanted ions $(\sim 230 \mathrm{~nm})$ as in previous studies on $\mathrm{Si}^{8-10)}$ The depth distributions of ions and the Frenkel pairs formed were calculated with a Monte Carlo code SRIM ${ }^{14)}$ (The Stopping and Range of Ions in Matter), as shown in Figs. 1(a) and (b).

Cross-section samples were prepared by the conventional method, as already reported in detail in Ref. 13): the irradiated sample was cut into half, and the two halves were pasted together face-to-face with epoxy glue. Then, this pasted slab was sectioned such that the damaged layers were visible, followed by dimpling and ion-milling according to standard sample preparation procedures for semiconductors and ceramics. ${ }^{15)}$

TEM and EELS were carried out using a Jeol JEM-200CX electron microscope equipped with Gatan Digi-PEELS model 766 and an ultrahigh-voltage transmission electron microscope (UHV-TEM), H-1250ST. 

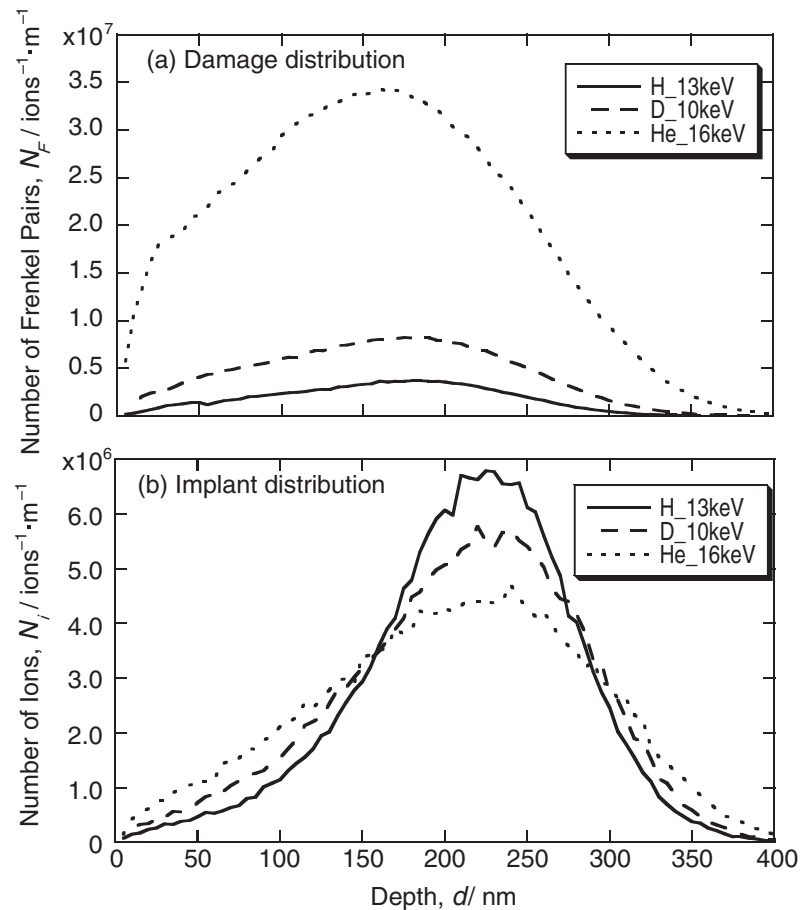

Fig. 1 SRIM code simulations for $\mathrm{Si}$ formed by $\mathrm{H}^{+}(13 \mathrm{keV}), \mathrm{D}^{+}(10 \mathrm{keV})$ and $\mathrm{He}^{+}(16 \mathrm{keV})$ irradiation. Depth distributions of (a) Frenkel pairs and (b) implanted ions.
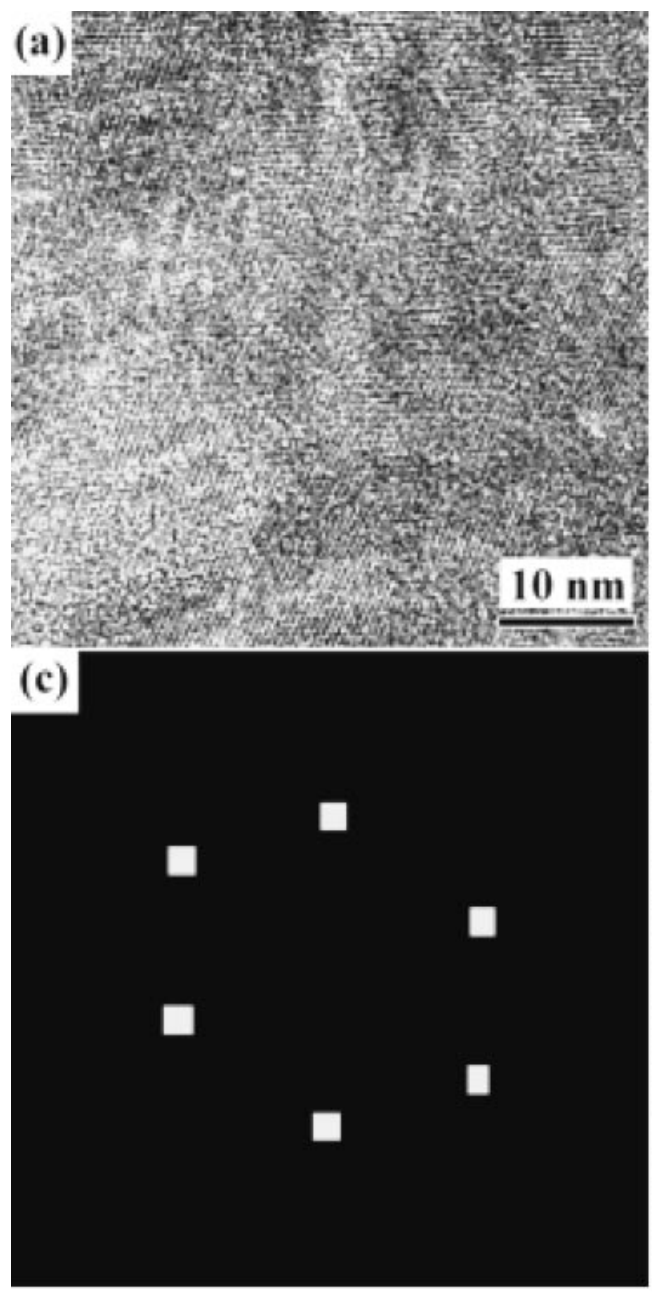

The mechanical properties of the irradiated samples were measured at room temperature with a CSIRO UMIS2000 ${ }^{\circledR}$ nanoindentation instrument equipped with a Berkovichshaped diamond indenter, in which the maximum penetration depth was approximately $100 \mathrm{~nm}$ (corresponding to a maximum load of $2-8 \mathrm{mN}$ ), to most effectively probe the damaged layer. Twenty measurements were conducted for each irradiation fluence. The data points obtained were used to construct load-displacement curves (L-D curves). Data points were averaged after eliminating those that showed some anomaly, presumably due to surface roughness. The details of the nanoindentation tests have already been reported in Ref. 16).

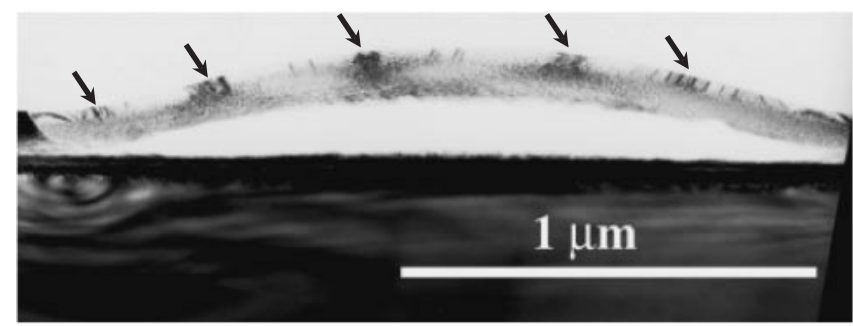

Fig. 2 XTEM image of a $\mathrm{H}$-blister after $\mathrm{H}^{+}$-irradiation at a dose of $1.0 \times 10^{22} \mathrm{~m}^{-2}$. Arrowheads indicate bend contours.
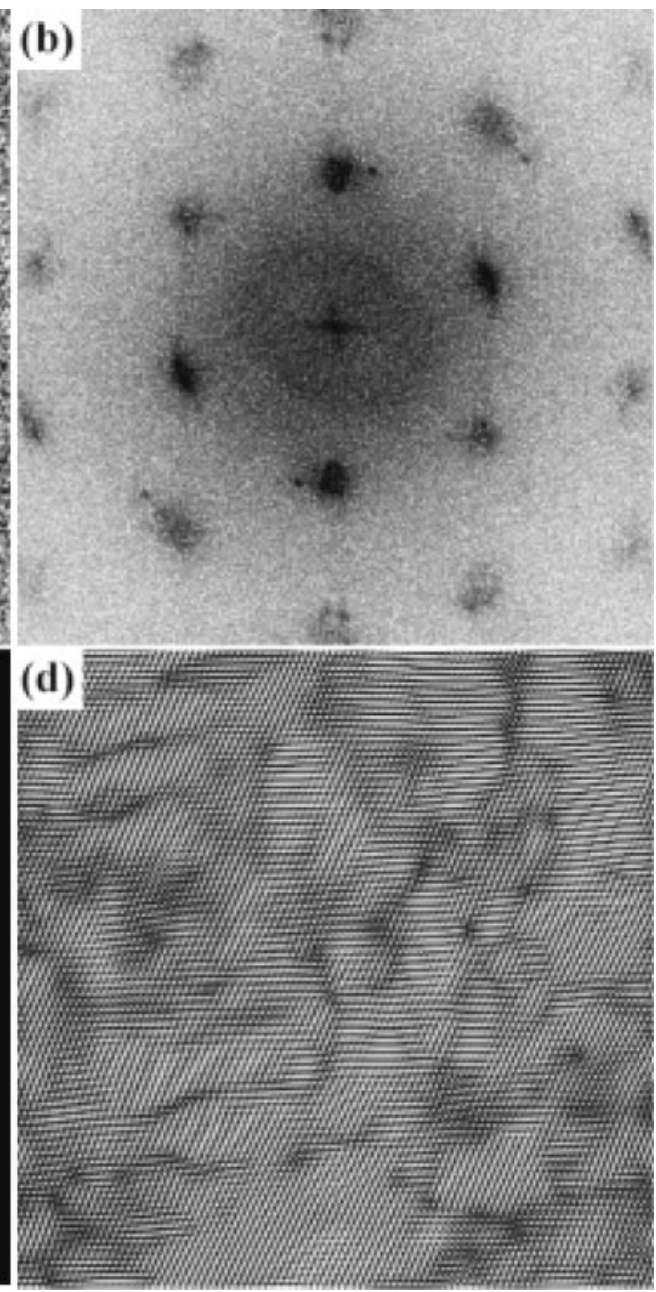

Fig. 3 (a) High-resolution lattice image of the lower defect-containing layer of H-blister skin. (b) FFT power spectrum of (a). (c) Mask to be applied to (b) for inverse FFT to eliminate noise and enhance the periodic structure. (d) Result of inverse FFT. 


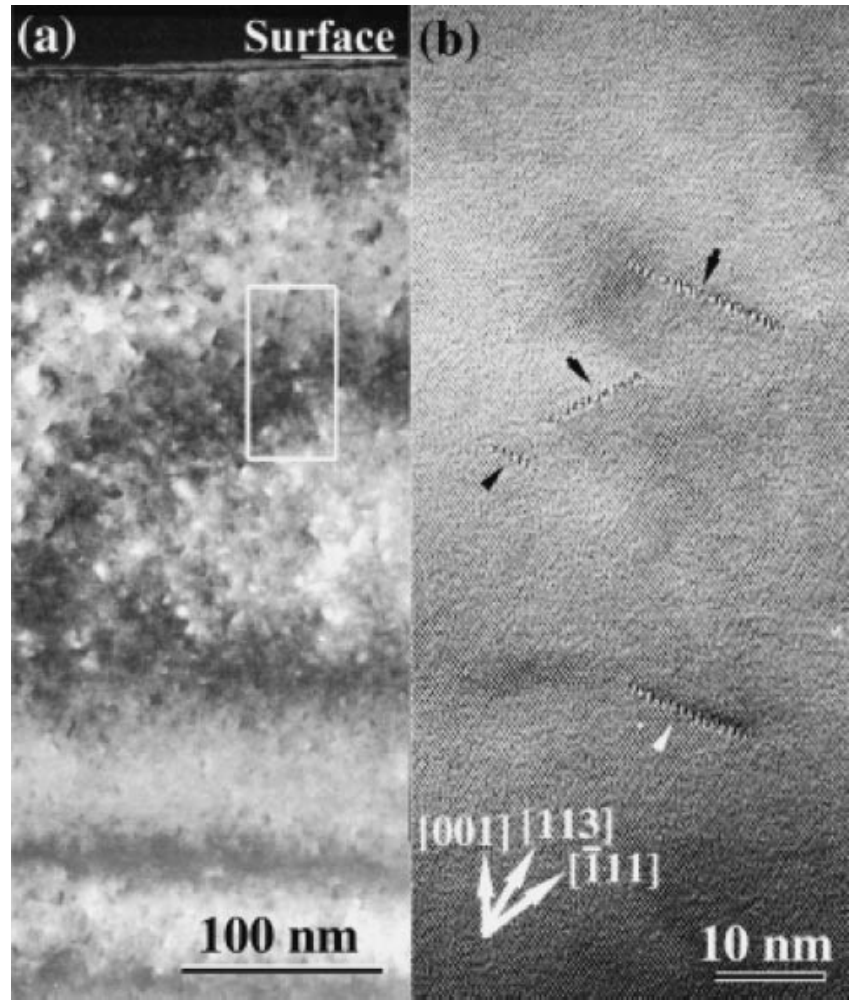

Fig. 4 (a) XTEM image of silicon $\mathrm{H}^{+}$-irradiated at a dose of $1.0 \times$ $10^{21} \mathrm{~m}^{-2}$. (b) High-resolution lattice image of the framed portion in (a). The black arrows indicate interstitial loops on the $\{311\}$ planes.

\section{Results}

\section{1 $\mathrm{H}^{+}$irradiation (H-blisters)}

An XTEM image of a H-blister (fluence $=1.0 \times$ $10^{22} \mathrm{~m}^{-2}$ ) is shown in Fig. 2. The skin thickness can be directly measured to be approximately $200 \mathrm{~nm}$, which coincides with the damage peak position in Fig. 1(a). This supports the gas-bubble network model for the blister formation, proposed in our previous reports.,10) The skin thickness is twice as large as that estimated by EELS in those reports. ${ }^{9,10)}$ This discrepancy will be discussed in Section 4.1. As mentioned in the introduction, the original crystal structure remains in the upper layer of the blister skin, which is best manifested by the bend contours at the top layer. These contours suggest that the top layer of the blister skin is elastically deformed. The inner wall contains a high density of defects and this part should bear most of the plastic deformation associated with blistering. ${ }^{17)}$

A high-resolution (HR) lattice image of this highly defective portion is shown in Fig. 3(a), and its fast Fourier transform (FFT) power spectrum in Fig. 3(b). Then, the mask shown in Fig. 3(c) was applied to the power spectrum, followed by the inverse FFT, to highlight the lattice fringes, as shown in Fig. 3(d). It is seen in the figure that the lattice fringes form domain-like microstructures up to $10 \mathrm{~nm}$ in size, the inter-domain boundaries between which exhibit lattice mismatch (interfacial dislocations) and/or bent lattice fringes, suggesting that the crystal orientation shows local fluctuation. One can estimate the orientational fluctuation of the fragmented domains from the spot broadening in

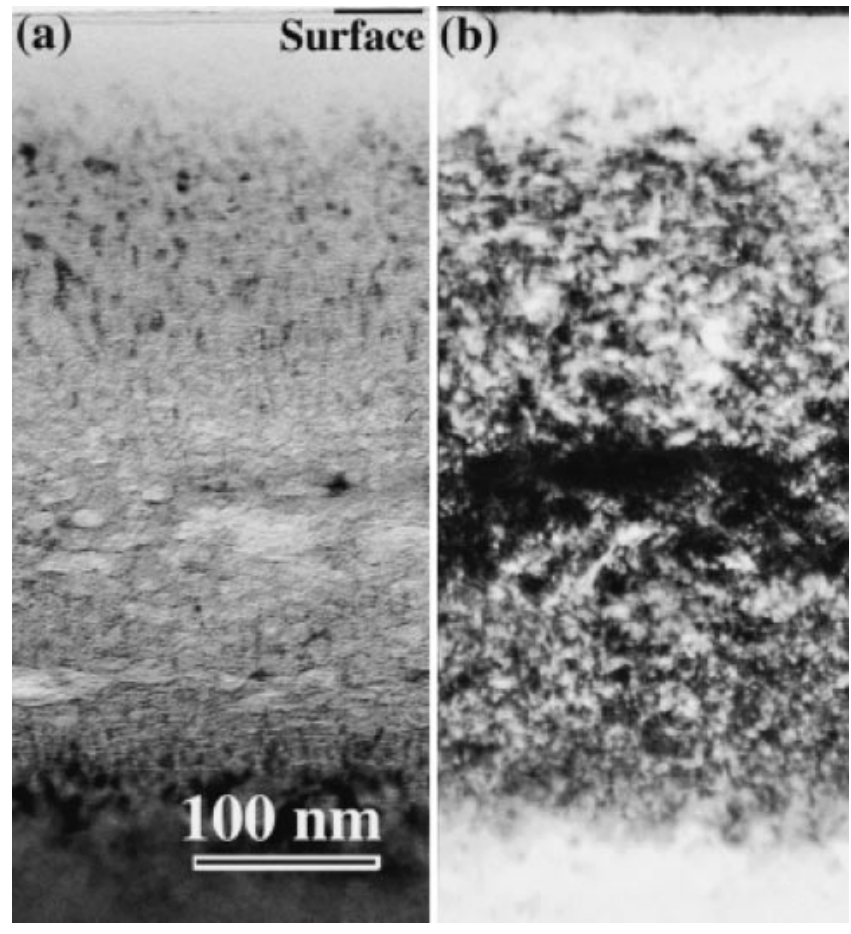

Fig. 5 Bright-field (a) and dark-field (b) XTEM images of silicon $\mathrm{H}^{+}$irradiated at a dose of $6.0 \times 10^{21} \mathrm{~m}^{-2}$.

Fig. 3(b) to be about $\pm 10^{\circ}$.

XTEM images for an irradiation dose of $3.0 \times 10^{21}$ and $6.0 \times 10^{21} \mathrm{~m}^{-2}$, which are below the threshold dose for blistering, are shown in Figs. 4 and 5, respectively, showing the evolution of internal structure prior to blistering. Figure 4(a) is a weak-beam dark-field image and (b) is an HRTEM image of the portion indicated in (a). It is seen that most of the small defects in (a) are interstitial faulted loops on the $\{311\}$ planes, which are well known to be formed by energetic particle (electron, neutron, ion) irradiation at elevated temperatures, where self-interstitial atoms are mobile $(>400 \mathrm{~K}){ }^{18-22)}$ This result indicates that the $\{311\}$ loops can be formed even at room temperature in the presence of a very high density of hydrogen. The $\mathrm{H}$ concentration was estimated to be approximately 30 at $\%$ around the projected range peak in Fig. 1(b) at a dose of $3.0 \times 10^{21} \mathrm{~m}^{-2}$.

Figure 5(a) was taken under a kinematical diffraction condition, letting the low index reflections deliberately deviate from their Bragg conditions. At this dose, thin large bubbles are observed around the depth of the projected range peak. The weak-beam dark-field image [Fig. 5(b)] shows the bubbles coalesce into cracks, and a high density of defects are dispersed over the region from the surface to the end-ofrange. It is also noted that another type of extended defects is observed in Fig. 5; these defects are seen as thin lines, extending roughly along the (001) planes and distributed on both sides of the bubble-distributing depth. These planar defects are thought to be hydrogen platelets or plate-like bubbles because the defects show a bright amplitude contrast under the kinematical diffraction condition [see Fig. 5(a)], which suggests that the projected thickness is less along the defects. It has been known ${ }^{23)}$ that the shape and orientation of 


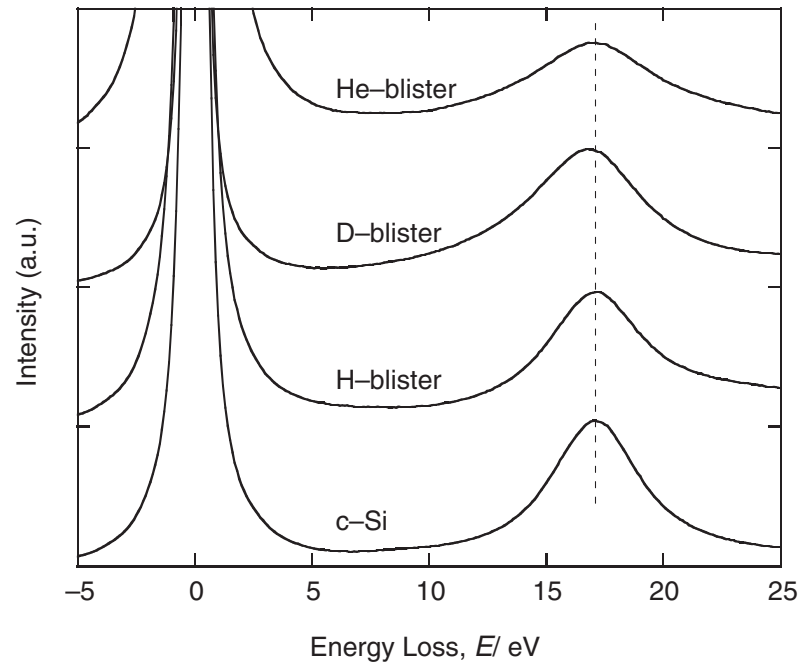

Fig. 6 EEL spectra taken of the low-loss region of H-, D-, and He-blister skins. A spectrum for crystalline silicon (c-Si) is also shown for comparison.

coherent precipitates in cubic metals and alloys are determined by the elastic anisotropy of the matrix: when the internal pressure is hydrostatic and the inequality $C^{\prime}(=$ $\left.\left(C_{11}-C_{12}\right) / 2\right)<C_{44}$ holds between the elastic constants of the matrix, the precipitate shape that is elastically most stable is the disc on the $\{001\}$ plane. On the other hand, if $C^{\prime}>C_{44}$, the most stable shape is needle-like extending along the $\langle 111\rangle$ direction. In the case of $\mathrm{Si}, C^{\prime}<C_{44}$, and thus, the fact that the bubbles extend parallel to the $\{001\}$ planes is not surprising. Moreover, the broken bonds along the $\{001\}$ crack plane are assumed to be terminated by hydrogen. Thus, the elastic strain energy dominates the surface energy inside the bubbles, which renders them even more effective in stabilizing the defect planes on the $\{001\}$. The present results indicate that blistering starts with the formation of extended defects, followed by the formation and evolution of bubbles. Eventually, large cracks propagate in the worst damaged layer by the coalescence of bubbles parallel to the (001) planes, which is consistent with the gas-bubble network model. $^{24)}$

EELS spectra of plasmon loss obtained from the blister skins are shown in Fig. 6, along with those acquired from a crystalline silicon sample for comparison. The plasmon peak from the H-blister skin shows a larger FWHM, reflecting the structural disorder, while the peak position remains almost unchanged, compared to that of crystalline $\mathrm{Si}$.

\section{2 $\mathrm{D}^{+}$irradiation (D-blisters)}

An XTEM image of a D-blister (fluence $=1.0 \times$ $10^{22} \mathrm{~m}^{-2}$ ) is shown in Fig. 7(a), where the blister skin is amorphous. A selected area diffraction (SAD) pattern [Fig. 7(b)] from the whole area in Fig. 7(a) shows the Bragg reflections from the crystalline substrate and halo rings from the amorphous blister skins. It is noted that the FWHMs of the halo rings seem rather small compared to those of typical amorphous silicon, e.g., silicon grown by PVD (plasma vapor deposition). The characteristic coherent size of the amorphous silicon was estimated to be approximately $1.2 \mathrm{~nm}$ from the FWHM of the halo rings, while that of the He-blister skin

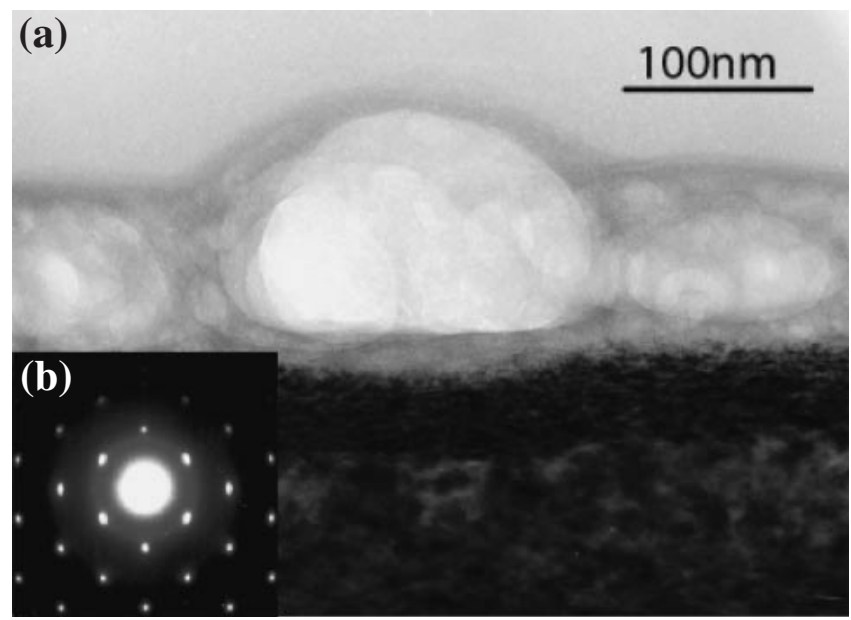

Fig. 7 XTEM image of D-blister after $\mathrm{D}^{+}$-irradiation at a dose of $1.0 \times 10^{22} \mathrm{~m}^{-2}$ (a) and corresponding electron diffraction pattern (b) from the whole area in (a).

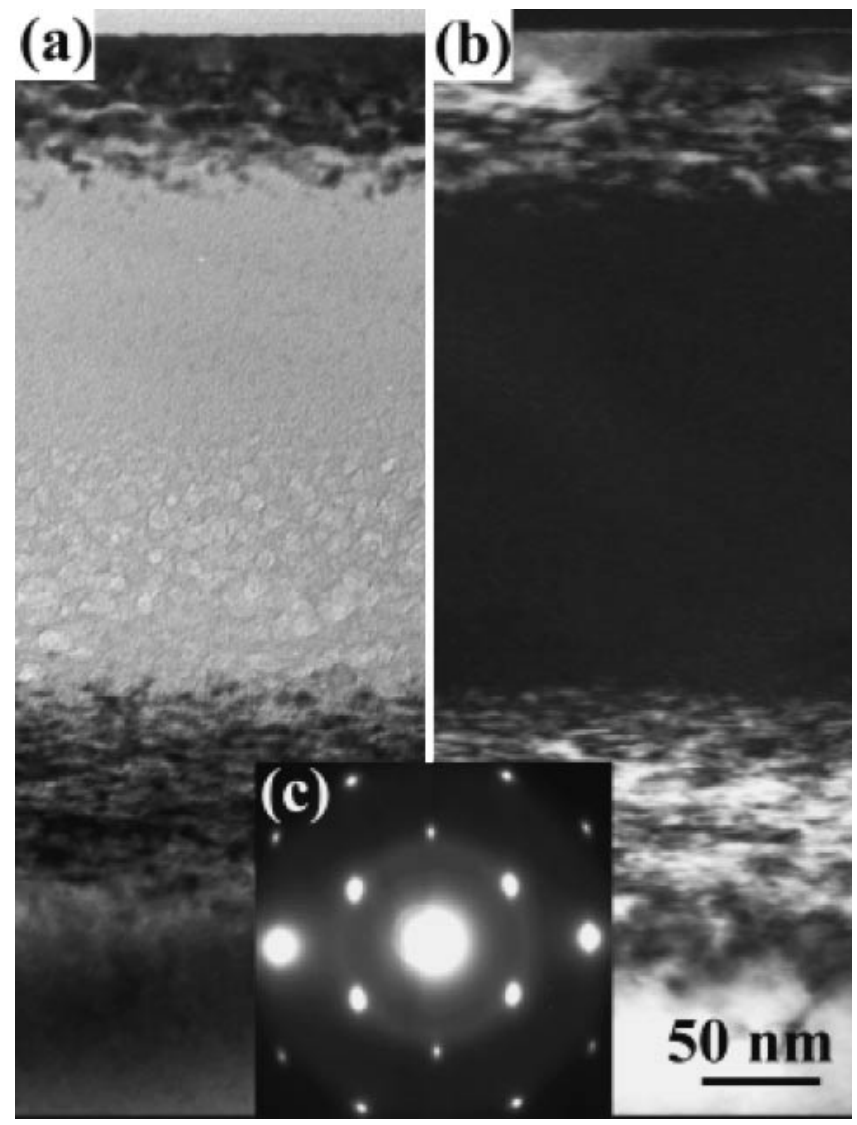

Fig. 8 (a) Bright-field XTEM image of Si irradiated by $\mathrm{D}^{+}$at a dose of $5.0 \times 10^{21} \mathrm{~m}^{-2}$. (b) Dark-field image of the same area as (a), taken with the 111 reflection strongly excited. (c) Electron diffraction pattern from the whole area in (a).

presented below was less than $0.8 \mathrm{~nm}$.

The pre-blistering stage shows that the amorphous layer forms where the induced damage exceeds 1 dpa $(\sim 1.0 \times$ $10^{21} \mathrm{~m}^{-2}$ ), and that bubbles are distributed along the peak depth of the projected range of the implanted ions, as shown in Fig. 8(a). The dark-field image of the same area [Fig. 8(b)] clearly shows high densities of extended defects on both sides 


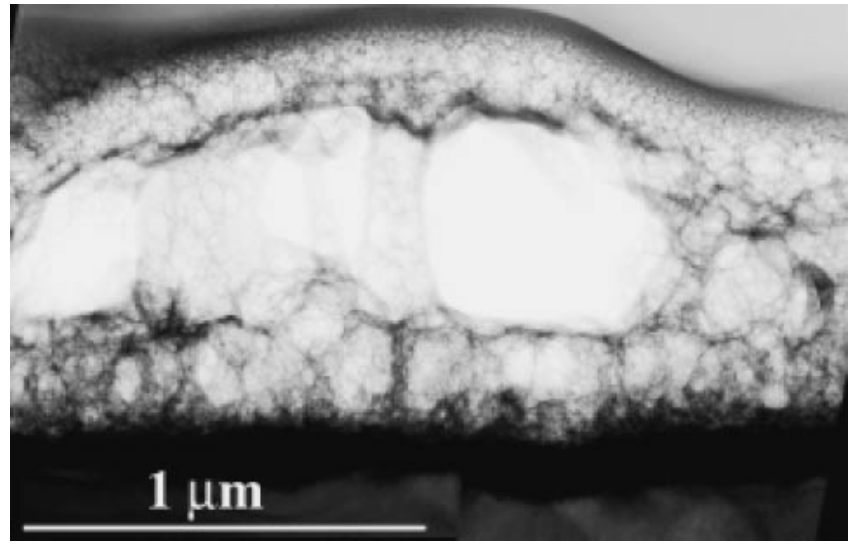

Fig. 9 XTEM image of a He-blister after $\mathrm{He}^{+}$-irradiation at a dose of $2.0 \times$ $10^{22} \mathrm{~m}^{-2}$.

of the amorphous layer, suggesting that amorphization proceeds by accumulation of such defect clusters. The FWHM of the halo rings in the SAD pattern is very similar to that of Fig. 7(b). The internal structure and the defect clusters formed before amorphization for fluences below $1 \times 10^{21} \mathrm{~m}^{-2}$ closely resemble those of $\mathrm{H}$-irradiation.

\section{3 $\mathrm{He}^{+}$irradiation (He-blisters)}

The internal structure of a He-blister (fluence $=2.0 \times$ $10^{22} \mathrm{~m}^{-2}$ ) is shown in Fig. 9. As already reported in our previous papers, ${ }^{9,10)}$ the blister wall is amorphous and includes a high density of He bubbles, whose size presumably corresponds to the gas distribution.

The $\mathrm{Si}$ matrix is amorphized by $\mathrm{He}^{+}$irradiation with a few tens of $\mathrm{keV}$ at a dose of about $1 \times 10^{21} \mathrm{~m}^{-2}$. The internal structures obtained at doses lower and higher than the amorphization dose are shown in Figs. 10(a) and (b), respectively. When the matrix is still crystalline [Fig. 10(a)], small defect clusters and extended defects are observed. The plane in which the extended defects lie is slightly deviated from the $\{001\}$ plane, though high-resolution observation reveals a rather diffuse and ill-defined structure, instead of $\{111\}$ - or $\{311\}$-type interstitial loops, whose contrast resemble those of $\mathrm{H}^{-}$or He-platelets. ${ }^{25,26)}$ Hence, the extended defects are thought to be He bubbles as well, lying in a plane that fluctuates around the $\{001\}$ plane, as described in Section 3.1. After the matrix is amorphized [Fig. 10(b)], the matrix no longer exhibits elastic anisotropy. Thus, the bubbles are more spherical as shown in the figure.

\section{Discussion}

\subsection{Discrepancy between the skin thickness values estimated by GIEM-EELS and XTEM}

In previous papers, we estimated the skin thickness of the blisters using GIEM and EELS.8,9) These estimates were nearly two to three times lower than the value expected by the gas-bubble network model, particularly at the top of the blister in H(D)-blisters. By contrast, the present direct observations of the blisters' cross-sections showed a skin thickness nearly equal to the damage peak depth. To explain this discrepancy, let us reexamine the principle behind

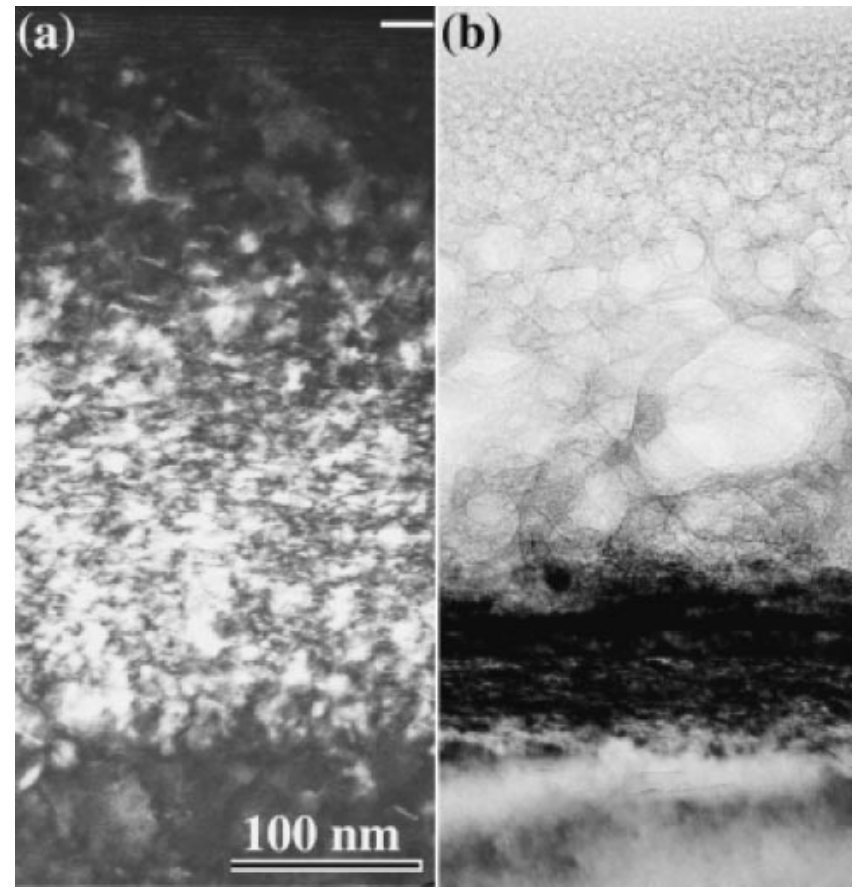

Fig. 10 Dark-field (a) and bright-field (b) XTEM images of silicon $\mathrm{He}^{+}-$ irradiated at a dose of $5.0 \times 10^{20} \mathrm{~m}^{-2}$ and $5.0 \times 10^{21} \mathrm{~m}^{-2}$, respectively.

thickness estimation by EELS. EELS intensity varies with the sample thickness, $t$, according to the following formula: ${ }^{27,28)}$

$$
t / \lambda=\ln \left(I_{t} / I_{0}\right)
$$

where $I_{0}$ is the intensity of elastically scattered electrons, $I_{t}$ the total electron intensity, and $\lambda$ the mean free path for inelastic scattering. Equation (1) indicates that the projected thickness $t$ is always obtained in units of $\lambda . \lambda$ is given by: ${ }^{27,28)}$

$$
\lambda=\frac{1}{n_{\mathrm{a}} \sigma_{\mathrm{p}}},
$$

where $n_{\mathrm{a}}$ is the atomic density and $\sigma_{\mathrm{p}}$ is the cross-section for plasmon excitation. The energy-loss spectra were recorded with an angle-limiting aperture that accepts only a fraction of the electrons. $\lambda$ depends on the atomic density, though it does not vary by more than a few $\%$ with ion irradiation. Hence, the discrepancy could be ascribed to an inaccurate estimation of $\sigma_{\mathrm{p}}$.

The differential cross-section for electrons inelastically scattered at an angle of $\theta$ by plasmon excitation can be approximated by: ${ }^{27,28)}$

$$
\frac{d \sigma}{d \Omega} \approx \frac{E_{\mathrm{p}}}{2 \pi a_{0} m_{0} v^{2} n_{\mathrm{a}}}\left(\frac{1}{\theta^{2}+\theta_{E}^{2}}\right)
$$

where $\theta_{E}\left(=E_{p} / \gamma m_{0} v^{2}\right)$ is the characteristic angle corresponding to the energy-loss $E_{\mathrm{p}}, a_{0}$ is the Bohr radius, $m_{0}$ is the electron mass, $v$ is the velocity of the electrons and $\gamma^{2}=\left(1-v^{2} / c^{2}\right)-1$. The total cross-section of the electrons inelastically scattered within $\theta_{1}-\theta_{2}$ can thus be calculated by integrating eq. (3) from $\theta_{1}$ to $\theta_{2}$,

$$
\sigma \equiv \int_{\theta_{1}}^{\theta_{2}} \frac{d \sigma}{d \Omega} d \Omega=\frac{E_{\mathrm{p}}}{2 a_{0} m_{0} v^{2} n_{\mathrm{a}}} \ln \frac{\theta_{E}^{2}+\theta_{2}^{2}}{\theta_{E}^{2}+\theta_{1}^{2}} .
$$


In GIEM-EELS measurements, the objective aperture (which acts as the angle-limiting aperture in the TEM mode) is located at a position slightly shifted from the incident beam in reciprocal space, unlike the conventional TEM-EELS measurements with $\theta_{1}=0$. The effective $\lambda$ in GIEM observations was thus estimated by substituting appropriate values compatible with the experimental conditions for all the constants in eq. (4) and by calculating $\lambda$ using eq. (2), which yielded $\lambda \approx 540 \mathrm{~nm}$. This gives a blister skin thickness roughly in agreement with that obtained by the present XTEM observation. In our previous papers, ${ }^{8,9)}$ we used the value of $\lambda=230 \mathrm{~nm}$ for $\mathrm{Si}$, and the aperture shift from the incident beam position was not taken into account. Therefore, the discrepancy is not attributed to the method itself, but rather to an inaccurate estimation of $\lambda$.

\subsection{Different morphology of blisters irradiated by hydrogen isotopes}

As already reported for the blisters in $\mathrm{SiC}$, morphologies of $\mathrm{He}$ - and $\mathrm{H}(\mathrm{D})$-blisters are very different, depending on whether the irradiating ions form chemical bonds with the matrix atoms. ${ }^{13}$ ) In the case of $\mathrm{Si}$, morphologies of $\mathrm{H}$ - and D-blisters are significantly different from each other, while He-blisters in Si exhibited a morphology very similar to that observed in $\mathrm{SiC}$. Hence, the blistering mechanism in He-blisters is common to $\mathrm{Si}$ and $\mathrm{SiC}$. Thus, the key factor is thought to be the structure of the target material at the onset of blistering: when the injected gas ions accumulate and start to form bubbles in the crystalline matrix, the gas pressure in the bubbles induces microcracks, which propagate and coalesce into large cracks around the depth of the projected range. This is actually the case for H-blisters, and hydrogen termination of the broken bonds enhances the crack formation and coalescence.

On the other hand, when the matrix is amorphous, the same blistering mechanism is at play in the case of D-blisters as in SiC. ${ }^{13)}$ The difference in blister morphology between $\mathrm{H}$ - and D-blisters is thus ascribed to the difference in mass number of $\mathrm{H}^{+}$and $\mathrm{D}^{+}$, since $\mathrm{D}^{+}$-irradiation amorphizes the Si matrix, while $\mathrm{H}^{+}$-irradiation does not bring about full amorphization of the Si matrix.

\subsection{Estimation of internal gas pressure}

The skin thickness and blister curvature revealed in the present XTEM observations enable us to estimate the internal gas pressure, which is essential for examining the blistering mechanism in terms of gas retention. We have already reported a rough estimation method, ${ }^{9}{ }^{\text {) }}$ where the elastic constants of the blister skins are assumed to be the same as those of the unirradiated crystal. However, in a recent study ${ }^{16)}$ that employed a combined method of nanoindentation testing and finite element analysis, we found that the Young's modulus, $E$, yield stress, $Y$, and hardness, $H$, of silicon vary significantly with the ion irradiation dose. Thus, we re-estimate the internal gas pressure of $\mathrm{H}$ - and D-blisters more precisely. Unfortunately, modelling the He-blister skin remains a difficult task because the skin contains a high density of gas bubbles and thus has a sponge-like structure, which prevents us from treating it as a simple continuum medium.

First, blister skins are modeled as a layered structure, where each layer has different mechanical properties according to the damage distribution. The mechanical parameters, $E, Y$, and $H$, have already been reported in Ref. 16). A bulk material divided into four layers according to Ref. 16) is constructed on a computer using the finite element method (FEM), in which cylindrical symmetry is assumed. Then, a thin opening is introduced at the depth corresponding to the blister skin thickness, followed by an increase in internal hydrostatic pressure until the upper layer is deformed to the observed blister shape. The internal pressure necessary to reproduce the blister's shape was $400 \mathrm{MPa}$ for both $\mathrm{H}$ - and Dblisters. Thus, the previous study ${ }^{9)}$ overestimated the internal pressures by nearly one order of magnitude. In the case of the $\mathrm{H}$-blister, the uppermost layer is elastically deformed due to its hardness and nearly $50 \%$ of the deformation is recovered by releasing the internal pressure. It was observed experimentally that a blister retained its shape even when the blister skin was perforated and the internal gas pressure was released. The deformation associated with blistering is hence plastic deformation, and irradiation presumably induces creep deformation. We do not take the creep effect into consideration in the present FEM simulation, but rather, increase the pressure until the final shape after stress release coincides with the observed shape. The resultant elastic and plastic strain distributions are shown in Figs. 11(a)-(c), in which only the largest strain components, $e_{11}$ 's, are shown. In the case of H-blisters, larger strains are concentrated along the periphery of the blister; particularly significant elastic strains are observed in the top layer, which retains high crystallinity. In the case of D-blisters, the elastic components (not shown) are negligibly small, and larger plastic deformations are observed around the top and outskirts of the blister.

Since the number densities and internal volumes of the blisters are already available in Ref. 9), the total numbers of gas atoms involved in the blisters can be estimated in the manner described in the literature: ${ }^{8,9,12)}$ only 14 and $49 \%$ of all implanted ions contributed to $\mathrm{H}$ - and D-blister formation, respectively; the rest remained as atomic hydrogen in the matrix.

Finally, it should be mentioned how such large, blisteringrelated plastic deformations occur in non-ductile covalent materials. A key factor is that the irradiated layer has an amorphous or finely-fragmented nano-crystalline structure. As discussed in Ref. 16), a system with no long-range structural order can be plastically deformed by rearranging the atomic configurations so as to accommodate the applied stress with the local average coordination and bond angles preserved. The deformation process macroscopically resembles creep deformation and is presumably enhanced by ion irradiation-induced diffusion.

\subsection{Small plasmon shift in blister skin}

As mentioned in Section 3.1-3, the plasmon energy of the blister skin remained almost unchanged with respect to that of crystalline silicon, whereas in $\mathrm{SiC}$, the plasmon energy was significantly reduced compared to the crystalline value. Plasmon energy is a function of the valence electron density $^{27,28)}$ and actually we linked the plasmon shift 

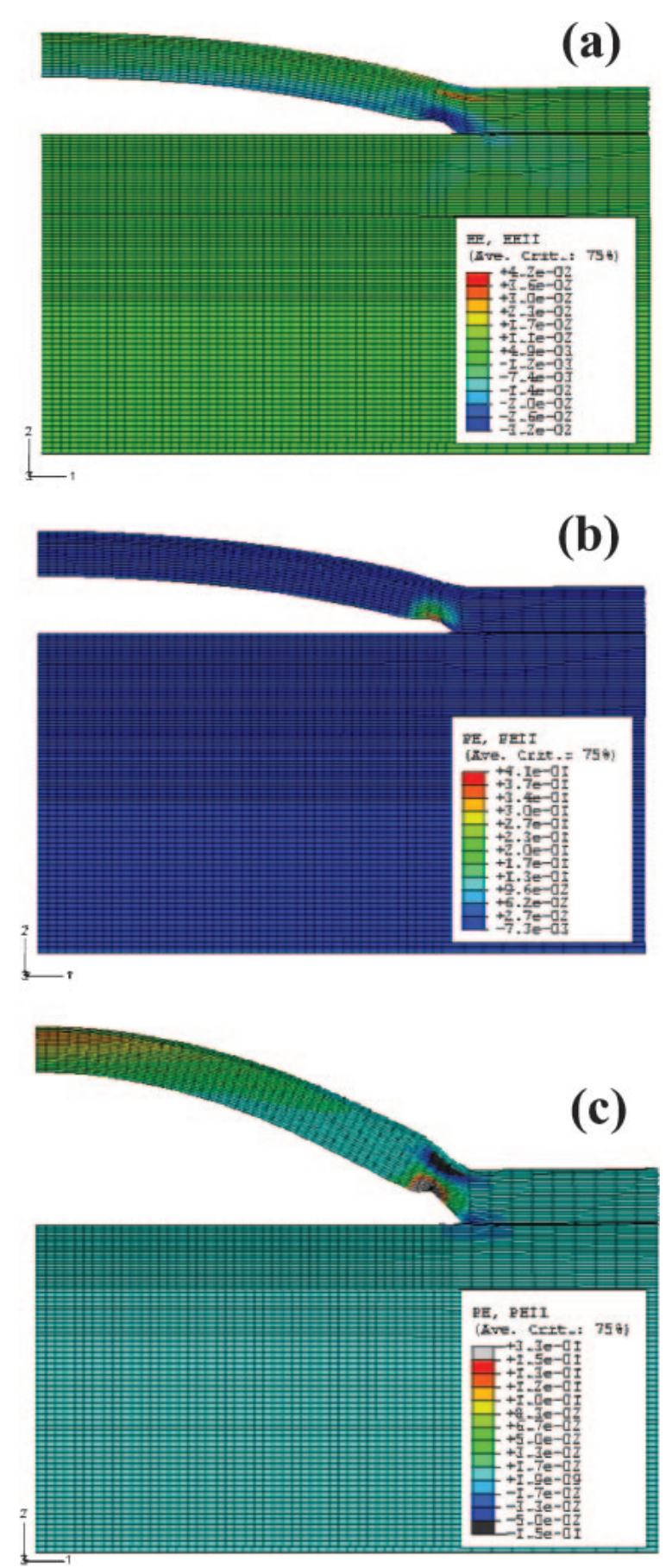

Fig. 11 Strain distributions in H- and D-blister skins, simulated with the finite element method based on the mechanical properties data in Ref. 16. (a) Elastic and (b) plastic strain components for a H-blister. (c) Plastic strain components for a D-blister.

in amorphous $\mathrm{SiC}$ due to electron- and ion-irradiation to irradiation-induced swelling. ${ }^{13,29)}$ The total swelling in electron- or neutron-irradiated $\mathrm{SiC}$ exceeded $10 \%,{ }^{29)}$ which was explained by typical void swelling (5-6\%) and asymmetric homo- and heteronuclear bond lengths (i.e., $\mathrm{C}-\mathrm{C} \mathrm{Si-Si}$, and $\mathrm{Si}-\mathrm{C}$ ) associated with amorphization of $\mathrm{SiC}^{29)}(7 \%)$.

In the case of $\mathrm{H}_{-}^{+}, \mathrm{D}^{+}-$and $\mathrm{He}^{+}$-irradiated $\mathrm{Si}$, the corresponding volume changes estimated from the plasmon shift were less than $2 \%$. Naturally, amorphization or structural disordering does not give rise to any asymmetric bond lengths in pure Si. Furthermore, the void swelling in $\mathrm{Si}$ associated with amorphization would presumably be very small since $\mathrm{Si}-\mathrm{Si}$ bonds are much more flexible than $\mathrm{Si}-\mathrm{C}$ and $\mathrm{C}-\mathrm{C}$ bonds in amorphous $\mathrm{SiC},{ }^{30)}$ and the induced strains could be relaxed to a great extent. The plasmon energies of CVD-grown amorphous $\mathrm{Si}$ and hydrogenated amorphous $\mathrm{Si}$ were indeed almost identical to that of crystalline $\mathrm{Si},{ }^{31)}$ which is consistent with the present results.

\section{Summary}

Cross-sectional structures of surface blisters and their precursors formed by $\mathrm{H}^{+}-, \mathrm{D}^{+}-$and $\mathrm{He}^{+}$-irradiation were examined by TEM. The results obtained can be summarized as follows:

(1) The blister skin of H-blisters consisted of a single crystalline upper layer ( $\sim 50 \mathrm{~nm}$ from the surface) and a highly defect-containing lower (inner) layer. In the inner layer, the crystal orientation fluctuated locally by about $\pm 10^{\circ}$.

(2) Below the critical dose for H-blistering, interstitial loops and planar bubbles formed on the $\{311\}$ and $\{100\}$ planes, respectively, over the region of high hydrogen density and damage distribution. At the damage peak depth, the bubbles coalesced into larger cracks, which would eventually induce blistering.

(3) Morphology of D-blisters is similar to those in $\mathrm{SiC}$, where bubbles form, grow and coalesce after the matrix is completely amorphized. The difference in morphology between $\mathrm{H}$ - and D-blisters stems from the fact that in the latter case, the matrix is amorphized before the bubbles start to form.

(4) He-blistering proceeded by amorphization of the irradiated layer, bubble formation and coalescence associated with $\mathrm{He}$ accumulation, followed by lifting up of the upper layer with increasing internal pressure. This He-blistering mechanism is common to most materials.

(5) The discrepancy between the blister skin thicknesses estimated by XTEM direct observation and GIEMEELS was explained by the correct estimation of the mean free path of inelastically scattered electrons.

(6) The internal gas pressure of $\mathrm{H}$ - and D-blisters was estimated by the finite element method, using measured mechanical properties of the blister skins. These results allowed us to estimate the gas fraction of the total amount of implanted ions actually involved in blister formation.

The present study, along with several of our other studies on blistering ${ }^{8-13,16,17)}$ have fully elucidated the evolution of substructures associated with blistering processes in covalent-bonded brittle materials.

\section{Acknowledgments}

This work is partly supported by Grants-in-Aid for Scientific Research (A) and (B) of JSPS, and for 21 century $\mathrm{COE}$ program (Isotope Science and Engineering from Basics to Applications) of MEXT. 


\section{REFERENCES}

1) D. J. Mazey: J. Nucl. Mater. 174 (1990) 196-209.

2) H. Verbeek and W. Eckstein: Application of Ion Beams to Metals (Plenum, New York 1974) p. 607.

3) J. Roth, R. Behrisch and B. M. U. Scherzer: J. Nucl. Mater. 53 (1974) 147-153.

4) M. Braun, B. Emmoth and J. L. Whitton: J. Nucl. Mater. 93-94 (1980) $728-733$.

5) N. Igata and A. Kohyama: J. Nucl. Mater. 103 (1981) 409-413.

6) G. Kohse and O. K. Harling: J. Nucl. Mater. 123 (1984) 1466-1469.

7) B. Constantinescu, C. Sarbu and L. Simionescu: Rad. Phys. Chem. 49 (1997) 411-414.

8) S. Muto, T. Matsui and T. Tanabe: Jpn. J. Appl. Phys. 39 (2000) 35553556.

9) S. Muto, T. Matsui and T. Tanabe: J. Nucl. Mater. 290-293 (2001) 131-135.

10) S. Igarashi, S. Muto, T. Tanabe and T. Maruyama: Mater. Trans., JIM 42 (2001) 2131-2132.

11) S. Igarashi, S. Muto, T. Tanabe, J. Aihara and K. Hojou: Surf. Coat. Technol. 158-159 (2002) 421-424.

12) S. Igarashi, S. Muto and T. Tanabe: J. Nucl. Mater. 307-311 (2002) 1126-1130.

13) S. Muto, T. Tanabe and T. Maruyama: Mater. Trans. 44 (2003) 2599_ 2604.

14) http://www.srim.org/SRIM/SRIM2003.htm
15) D. B. Williams and C. B. Carter: Transmission Electron Microscopy, (Plenum, New York 1996) pp. 165-166.

16) S. Nakano, S. Muto and T. Tanabe: J. Jpn. Inst. Met. (in Japanese), 69 (2005) 815-824. English version is to be published in Mater. Trans.

17) S. Muto, T. Tanabe and S. Igarashi: Phys. Scri. T108 (2004) 19-22.

18) C. A. Ferreira Lima and A. Howie: Philos. Mag. 34 (1976) 1057-1071.

19) A. Bourret: Inst. Phys. Ser. 87 (1987) 39-44.

20) I. G. Salisbury and M. H. Loretto: Philos. Mag. A 39 (1979) 317-323.

21) S. Takeda: Jpn. J. Appl. Phys. 30 (1991) L639-L642.

22) R. Oshima, T. Kawano and R. Fujimoto: J. Nucl. Mater. 212-215 (1994) 293-297.

23) N. Kinoshita and T. Mura: Phys. Soc. Solids 5(a) (1971) 759-756.

24) T. Katabuchi, N. Kawachi and Y. Tagishi: J. Appl. Phys. 86 (1999) 3030-3035.

25) S. Muto, S. Takeda and M. Hirata: Philos. Mag. A 2 (1995) 1057-1074.

26) S. Muto, S. Takeda and M. Hirata: Mater. Sci. Forum 196-201 (1995) 1171-1176.

27) R. F. Egerton, Electron Energy-Loss Spectroscopy in the Electron Microscope, (Plenum, New York, 1996) p. 154.

28) R. F. Egerton, Electron Energy-Loss Spectroscopy in the Electron Microscope, (Plenum, New York, 1996) p. 185.

29) S. Muto and T. Tanabe: J. Appl. Phys. 93 (2003) 3765-3775.

30) M. Kohyama: Philos. Mag. Lett. 79 (1999) 659-672.

31) S. Muto, Y. Kobayashi, K.-M. Yu, W., Walukiewicz, C. J. Echer, H.-C. Jin and J. R. Abelson: Proc. Int'l. Symp. on Solid-Solid Phase Transformations '99 (Kyoto, May, 1999), pp. 1231-1234. 